\title{
O professor como modelo aos seus estudantes: perspectivas da área da saúde
}

\author{
The teacher as a role model to its students: perspectives from the health area

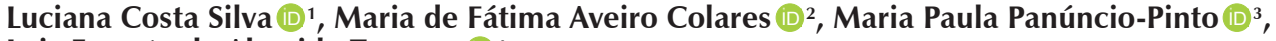 \\ Luiz Ernesto de Almeida Troncon $(\mathbb{D})^{4} \bowtie$ \\ 1 Programa de Pós-Graduação em Ciências da Saúde, Faculdade de Medicina de Ribeirão Preto, Universidade de São Paulo, \\ Departamento de Clínica Médica. Ribeirão Preto, São Paulo, Brasil. \\ 2 UNIFACEF-Centro Universitário Municipal de Franca, Departamento de Psicologia. Franca, São Paulo, Brasil. \\ ${ }^{3}$ Faculdade de Medicina de Ribeirão Preto, Universidade de São Paulo, Departamento de Ciências da Saúde e Centro de Desenvolvimento Docente \\ para o Ensino. Ribeirão Preto, São Paulo, Brasil. \\ ${ }^{4}$ Faculdade de Medicina de Ribeirão Preto, Universidade de São Paulo, Departamento de Clínica Médica e Centro de Desenvolvimento Docente \\ para o Ensino. Ribeirão Preto, São Paulo, Brasil.
}

Como citar este artigo (How to cite this article):

Silva LC, Colares MFA, Panúncio-Pinto MP, Troncon LEA, O professor como modelo aos seus estudantes: perspectivas da área da saúde (The teacher as a role model to its students: perspectives from the health area). Sci Med. 2019;29(4):e35862. https://doi.org/10.15448/19806108.2019.4.35862

\section{RESUMO}

A formação do estudante nas profissões da saúde envolve a incorporação de habilidades e competências gerais e específicas, adquiridas em experiências práticas sob a supervisão de professores ou preceptores, cujas características podem influenciar os estudantes e os guiar na construção de sua identidade profissional. Nesse contexto, define-se "modelo" (role model) como o profissional que serve de exemplo, por suas qualidades positivas, sendo imitado pelos estudantes, por demonstrar habilidades e características pessoais que os impressionam e inspiram. Modelos positivos exibem expertise profissional, boa comunicação e relacionamento com seus pacientes e com os estudantes, boas habilidades de ensino e, sobretudo, características pessoais como integridade, solidariedade e entusiasmo. Por outro lado, os estudantes são capazes de reconhecer atributos negativos indesejáveis, opostos ás características positivas. Nossos estudos sugerem que a percepção dos estudantes brasileiros sobre os modelos não difere do que é descrito no cenário internacional e que talvez não existam diferenças apreciáveis entre as várias profissões da saúde. Os professores e preceptores considerados pelos estudantes como bons modelos, surpreendentemente, desconhecem que exercem essa influência, mas têm visão semelhante aos dos estudantes sobre os atributos positivos de um bom modelo. Dada a importância dos modelos na formação pessoal e profissional na área da saúde, é imperioso que as escolas tomem medidas para dispor em seu corpo docente de predomínio de modelos positivos e para evitar que seus professores e preceptores emitam comportamentos que expressem qualidades negativas. Estas medidas envolvem atividades de desenvolvimento docente e valorização dos docentes por sua atuação no ensino. No entanto, essas medidas somente farão sentido se as escolas oferecerem aos seus docentes boas condições de trabalho e de remuneração e, sobretudo, tiverem cultura institucional que privilegie relações humanizadas entre os seus membros.

DESCRITORES: Tutores/educação, Docente/capacitação, Profissões em saúde, Estudantes.

\section{ABSTRACT}

Student education and training in the health professions involve acquisition of general and specific skills and competencies which happens throughout practical experiences under the supervision of teachers or clinical tutors. Supervisor characteristics may influence students and guide them in building their professional identity. In this context, a role model is defined as the professional whose positive qualities are likely to be imitated by the students, as they demonstrated skills and personal characteristics that impress and inspire them. Positive role models exhibit professional expertise, good communication and relationships with patients and students, good teaching skills and, most importantly, personal characteristics such as integrity, compassion and enthusiasm. On the other hand, students seem to be able to recognize undesirable negative attributes as opposed to the positive ones. Our studies suggest that the perception of Brazilian students on models attributes does not differ from what is described in the international literature and that there may not be substantial differences between students from the various health professions on their views. Teachers and preceptors who students consider to be good role models, surprisingly, are unaware that they have such influence, but have similar views as students about the positive attributes of a role model. Given the importance of role models in student personal and professional development, schools must take steps to have positive models prevailing in their faculty and to prevent clinical tutors from expressing behaviours that convey negative qualities. These measures involve teacher training and faculty development activities and appreciation of faculty members for their performance in teaching. However, these measures will only make sense if schools offer their teachers good working conditions and, above all, possess an institutional culture that favours humanized relationships within the academic community.

KEYWORDS: Role modelling, Health professions students, Clinical tutors, Faculty development. 


\section{INTRODUÇÃO}

A formação profissional na área da saúde é um processo complexo que envolve a aquisição e o desenvolvimento de habilidades e competências necessárias para realizar as ações essenciais de uma determinada atividade profissional [1-3]. A aquisição dessas competências envolve experiências em cenários de prática sob a supervisão de professores ou preceptores cujas características podem influenciar os estudantes e, sobretudo, guia-los na construção de sua identidade profissional $[4,5]$.

Nesse contexto da formação profissional em saúde, é possível definir "modelo" (role model) como o profissional que serve de exemplo aos estudantes pelas suas qualidades positivas, sendo imitado por eles por demonstrar habilidades e características pessoais que os impressionam e inspiram [4,6-8]. Essa influência se exerce de modo a facilitar ao estudante adquirir habilidades profissionais, criar sua identidade profissional e incorporar atributos do profissionalismo $[4,6-8]$.

Os modelos - professores, tutores e supervisores a quem os estudantes estão expostos - têm sido reconhecidos como uma das mais importantes influências na formação profissional $[1,6,7]$. A exposição a eles inspira os estudantes e contribui de forma decisiva para sua educação e seu desenvolvimento profissional e pessoal $[6,7,9]$. As interações do estudante com modelos positivos e o contato com seu entusiasmo pessoal e satisfação profissional podem impactar sua formação de muitas formas, influenciando, inclusive, a escolha da especialidade e dos rumos de carreira [7].

Existem evidências da importância de professores, supervisores e tutores tomarem consciência sobre o seu papel de modelos aos seus estudantes e da profunda influência que podem exercer na sua formação, o que os predisporia a fazer esforço intencional para melhor definir os aspectos que estariam "modelando" $[6,10,11]$.

Neste sentido, esse artigo tem como objetivos apresentar à comunidade educacional da área da saúde, conceitos básicos sobre a influência dos modelos, os atributos positivos que os definem, as características negativas que não deveriam ser expressas, a visão que os próprios modelos têm do seu papel e as maneiras como as instituições poderiam agir para dispor de predomínio de modelos positivos em seu corpo docente.

\section{Atributos positivos desejáveis}

Diferentes trabalhos com estudantes de Medicina relatam resultados semelhantes quanto à sua percepção das várias características dos professores ou supervisores que eles consideram bons modelos [7, 12-18]. Estes atributos se agrupam em diferentes domínios: 1) expertise profissional ("habilidades clínicas"); 2) relacionamento com pacientes e com estudantes; 3 ) habilidades de ensino e 4) características pessoais ou de personalidade do modelo [18]. É interessante notar que vários destes grupos de domínios incluem atributos que se superpõe, como empatia (com os pacientes e com os estudantes), habilidades de comunicação (com pacientes, estudantes, colegas de profissão e membros de outras profissões) e características de personalidade, como integridade, sinceridade, confiança, solidariedade e entusiasmo (importantes em qualquer atividade profissional) [13].

Em estudo recente com estudantes de Terapia Ocupacional, verificamos que sua percepção não difere muito daquela dos estudantes de Medicina, sugerindo, assim, que talvez os modelos sejam vistos de modo semelhante nas várias profissões da saúde [8]. Em nosso estudo, em que parte importante da pesquisa foi de natureza qualitativa, a análise de conteúdo deu origem a três categorias principais, nas quais foram identificados atributos que as compõem (subcategorias). As categorias principais foram: 1) relacionamento interpessoal (estudante e paciente), em que os atributos mais citados foram empatia, bom relacionamento com estudantes e pacientes e boa comunicação; 2) habilidades para ensinar, contendo os atributos: didática, criatividade e gostar de ensinar; 3 ) profissionalismo, em que os atributos de organização, ética e base teórica (conhecimento) foram os mais apontados.

\section{Atributos negativos indesejáveis}

Ao contrário das características positivas dos modelos, os atributos negativos são uma questão pouco estudada na caracterização dos modelos profissionais. Porém, ao menos um estudo com estudantes de Medicina discute que a influência dos professores ou supervisores com esses atributos negativos pode ser mais forte do que os modelos positivos, uma vez que eles podem desmotivar os estudantes [19].

Em princípio, os atributos negativos podem enquadrar-se nas mesmas categorias que os positivos, mas tendo significado oposto: 1) expertise profissional (falta de conhecimentos ou desatualização); 2) relacionamento com pacientes e com estudantes (falta de empatia, desrespeito); 3) habilidades de ensino (desorganização ou ensino desestruturado; não prover devolutiva aos estudantes) e 4) características pessoais 
ou de personalidade do modelo (arrogância, falta de entusiasmo pela profissão ou desinteresse naquilo que faz) [18].

Em estudos que realizamos com estudantes dos cursos de Medicina [20] e de Terapia Ocupacional [8] encontramos achados semelhantes aos descritos na literatura, o que indica que a percepção dos estudantes brasileiros não difere do que é descrito no cenário internacional e que talvez não existam diferenças apreciáveis entre os estudantes das várias profissões da saúde.

\section{A visão dos próprios modelos}

Em estudo em que foram entrevistados médicos (professores e preceptores) que haviam sido indicados por estudantes de Medicina como sendo bons modelos [20], as principais características que foram destacadas por eles, como possíveis explicações para o fato de terem sido indicados, foram: habilidade de comunicação empática com pacientes e com os próprios estudantes, respeito e estabelecimento de bom vínculo com eles; raciocínio clínico apurado e vivências clínicas suficientes para poder ensinar, preocupação com o ensino e prazer em ensinar, além de atributos pessoais como idealismo, otimismo, humildade, espontaneidade, e reconhecimento de seus limites. Estes atributos são também descritos na literatura internacional [7].

Questionados sobre os meios de aquisição dessas características positivas, os modelos invariavelmente assinalaram também possuir em seu contexto de formação pessoal e profissional a inspiração de outros modelos, ou seja, profissionais que os estimularam a ser como são. Foram citados pessoas, sejam familiares ou professores, a que foram expostos durante a sua formação, que lhes passaram valores morais e éticos que foram importantes para estabelecer a forma como exercem tanto a profissão médica como a docência.

Neste estudo [20], foi marcante o fato de os professores e preceptores indicados como modelos desconhecerem totalmente que exerciam essa influência sobre os estudantes, bem como não terem conhecimento das razões que levaram a essa indicação. Isso reforça a necessidade de maior valorização pelas instituições da importância do papel dos modelos, bem como de melhor preparar professores e tutores para, com seu exemplo, influenciar efetiva e positivamente os estudantes na aquisição das competências necessárias e, sobretudo, na construção da sua identidade profissional [21].

\section{Recomendações para as instituições}

A inequívoca importância que o papel de modelo dos professores tem para a formação dos estudantes torna pertinente a preocupação das instituições em contar com corpo docente onde predominem os "bons modelos". Para isso seria necessário conscientizar dirigentes e professores da importância dos bons modelos e sobre as características que os definem. Essa conscientização envolveria também instruí-los sobre o que os estudantes consideram como características negativas (atributos dos “anti-modelos"), e capacitá-los para evitar ao máximo a emissão de comportamentos que expressassem estas qualidades negativas. Essa conscientização e o investimento comportamental caberiam em atividades específicas de desenvolvimento docente convencional, mas poderia ser mais produtiva a orientação dos professores mais novos, por certo tempo, por mentores, docentes mais experientes e mais familiarizados com a cultura institucional [22]. Nesse sentido, convêm lembrar que um dos padrões de qualidade internacional das escolas inclui dispor de programas de desenvolvimento docente, junto com políticas de valorização profissional [23]. Para os cursos de Medicina brasileiros, as Diretrizes Curriculares Nacionais (artigo 34), recomendam a existência de programas permanentes de formação e desenvolvimento docente [24].

A manutenção pelas instituições de programas de valorização e promoção dos docentes com base em sua atuação no ensino deveria contar com indicadores que expressassem serem eles bons modelos para o estudante. As evidências disso poderiam ser, por exemplo, testemunhos de estudantes ou convites para participação em atividades extracurriculares da iniciativa dos estudantes, apresentadas no portfólio educacional do professor. A eventual adoção desse instrumento na cultura profissional docente nacional facilitaria, também, que, na admissão, pudesse ser analisado se o(a) candidato(a) apresenta evidências de ter sido um bom modelo para os estudantes, ou de considerar isso como importante para sua atuação docente.

Claro está que estas medidas somente farão sentido se as escolas oferecerem ao seu corpo docente boas condições de trabalho e de remuneração e, sobretudo, tiverem cultura institucional que privilegie relações humanizadas entre os seus membros.

\section{NOTAS}

\section{Apoio financeiro}

Este estudo não recebeu apoio financeiro de fontes externas. 
Declaração de conflito de interesses

Os autores declaram não haver conflitos de interesses relevantes ao conteúdo deste estudo.

\section{Contribuições dos autores}

Todos os autores fizeram contribuições substanciais para concepção, ou delineamento, ou aquisição, ou análise ou inter- pretação de dados; e redação do trabalho ou revisão crítica; e aprovação final da versão para publicação.

Disponibilidade dos dados e responsabilidade pelos resultados

Todos os autores declaram ter tido total acesso aos dados obtidos e assumem completa responsabilidade pela integridade destes resultados.

\section{REFERÊNCIAS}

1. Mann KV. Theoretical perspectives in medical education: past experience and future possibilities. Med Educ. 2011;45(1):60-8. http://dx.doi.org/10.1111/j.1365-2923.2010.03757.x

2. Silva RF, Aquilante AG, Zem-Mascarenhas SH, Kishi RGB, Varga CRV. Análise das situações simuladas da prática médica. Rev Bras Educ Méd. 2012;36(1):55-62. http://dx.doi.org/10.1590/S0100-55022012000400008

3. Cotta RMM, Costa GD, Mendonça ET. Portfólio reflexivo: uma proposta de ensino e aprendizagem orientada por competências. Cien Saude Colet. 2013;18(6):1847-56. http://dx.doi.org/10.1590/S1413-81232013000600035

4. Tan CP, Van der Molen HT, Schmidt HG. A measure of professional identity development for professional education. Studies in Higher Education. 2017;42(8):1504-19. https://doi.org/10.1080/03075079.2015.1111322

5. Cruess RL, Cruess SR, Boudreau JD, Snell L, Steinert Y. Reframing medical education to support professional identity formation. Acad Med. 2014;89(11):1446-51. http://dx.doi.org/10.1097/ACM.0000000000000427

6. Passi V, Johnson N. The impact of positive doctor role modeling. Med Teach. 2016;38(11):1139-45. http://dx.doi.org/ 10.3109/0142159X.2016.1170780

7. Passi V, Johnson S, Peile E, Wright S, Hafferty F, Johnson N. Doctor role modeling in medical education: BEME Guide No. 27. Med Teach. 2013;35(9):e1422-36. http://dx.doi.org/10.3109/0142159X.2013.806982

8. Silva LC, Troncon LEA, Panúncio-Pinto MP. Perceptions of occupational therapy students and clinical tutors on the attributes of a good role model. Scand J Occup Ther. 2019; 26 (4): 283-293. http://dx.doi.org/10.1080/11038128.2018. 1508495

9. Kapila D. Female role models in medicine: a medical student's perspective. J Adv Med Educ Prof. 2018; 6 (1): 49-50.

10. Cruess SR, Cruess RL, Steinert Y. Role modeling: making the most of a powerful teaching strategy. BMJ. 2008;336(7646):718-21. http://dx.doi.org/10.1136/bmj.39503.757847.BE

11. Jack K, Hamshire C, Chambers A. The influence of role models in undergraduate nurse education. J Clin Nurs. 2017;26(23-24):4707-15. http://dx.doi.org/10.1111/jocn.13822

12. Wright S. Examining what residents look for in their role models. Acad Med. 1996;71(3):2902. http://dx.doi. org/10.1097/00001888-199603000-00024

13. Elzubeir MA, Rizk DE. Identifying characteristics that students, interns and residents look for in their role models. Med Educ. 2001;35(3):272-77. http://dx.doi.org/10.1046/j.1365-2923.2001.00870.x

14. Aguilar A, Stupans I, Scutter S, King S. Exploring professionalism: the professional values of Australian occupational therapists. Aust. Occup Ther J. 2012;59(3);209-17. http://dx.doi.org/10.1111/j.1440-1630.2012.00996.x

15. Althouse LA, Stritter FT, Steiner BD. Attitudes and approaches of influential role models in clinical education. Adv Health Sci Educ. 1999;4(2):111-22. http://dx.doi.org/10.1023/A:1009768526142

16. Cote L, Leclere H. How clinical teachers perceive the doctor-patient relationship and themselves as role models. Acad Med. 2000;75(11):1117-24. http://dx.doi.org/10.1097/00001888-200011000-00020

17. Weissmann PF, Branch WT, Gracey CF, Haidet P, Frankel RM. Role modeling humanistic behaviour: learning bedside manner from the experts. Acad Med. 2006;81(7):661-67. http://dx.doi.org/10.1097/01.ACM.0000232423. 81299.fe 
18. Burgess A, Goulston K, Oates K. Role modelling of clinical tutors: a focus group study among medical students. BMC Med Educ. 2015;15:17. http://dx.doi.org/10.1186/s12909-015-0303-8

19. Murakami M, Kawabata H, Maezawa M. The perception of the hidden curriculum on medical education: an exploratory study. Asia Pac Fam Med. 2009;8(1):9. http://dx.doi.org/10.1186/1447-056X-8-9

20. Colares MFA. O professor de medicina como modelo profissional: a visão de estudantes e professores [relatório final de estágio de pós-doutorado]. Ribeirão Preto: Universidade de São Paulo; 2017. https://doi.org/10.11606/issn.21767262.v41i4p516-570

21. Paice E, Heard S, Moss F. How important are role models in making good doctors? BMJ. 2002;325(7366):707-10. http://dx.doi.org/10.1136/bmj.325.7366.707

22. Steinert Y, Mann KV. Faculty development: principles and practices. J Vet Med Educ. 2006;33(3):317-24. https://doi.org/10.3138/jvme.33.3.317

23. World Federation of Medical Education. Basic medical education WFME global standards for quality improvement [Internet]. Ferney-Voltaire: WFME; 2015 [capturado em 07 nov. 2019]. Disponível em: https://wfme.org/standards/ bme/. https://doi.org/10.1046/j.1365-2923.1998.00189.x

24. Brasil. Ministério da Educação. Conselho Nacional de Educação. Câmara de Educação Superior. Resolução no 3, de 20 de junho de 2014. Institui Diretrizes Curriculares Nacionais do Curso de Graduação em Medicina e dá outras providências [Intenet]. Brasília: Ministério da Educação; 2014 [capturado em 07 nov. 2019]. Disponível em: http:// portal.mec.gov.br/index.php?option=com_docman\&view=download\&alias $=15874-$ rces003-14\&category_slug= junho-2014-pdf\&Itemid=30192. https://doi.org/10.1590/s1413-24782012000100012 